\title{
The Princeton FRC Rotating-Magnetic-Field-Experiment RF System*
}

\author{
C. Brunkhorst, B. Berlinger, N. Ferraro, S.A. Cohen \\ Princeton Plasma Physics Laboratory, Princeton University, Princeton, NJ
}

\begin{abstract}
The Princeton FRC (PFRC) is a compact device that utilizes odd-parity[1] radio-frequency rotating magnetic fields $\left(\mathrm{RMF}_{\mathrm{o}}\right)$ to heat electrons[2] and drive azimuthal current in a cylindrical plasma column. A serendipity discovery is that the RF system serves as a useful diagnostic tool, providing data on RF-field penetration into the plasma.[3] Efficient use of RF power is required to accommodate the small size of the device and for its development into a practical fusion reactor. The RF system is described along with its evolution and lessons learned.
\end{abstract}

\section{THE PRINCETON FRC EXPERIMENT}

The central section of the PFRC vacuum vessel is a multi-port Pyrex pipe $81-\mathrm{cm}$ long by $10-\mathrm{cm}$ ID. Rotatingmagnetic-field coils (RFCs) are placed symmetrically outside the vessel. Inside the vessel are 10 flux conservers, coaxial copper rings of $8-\mathrm{cm}$ ID whose primary function is to slow the radial expansion of the $\mathrm{RMF}_{\mathrm{o}}$-formed plasma. Diagnostics, $\mathrm{RMF}_{0}$ coils and $\mathrm{RF}$ matching circuits must all fit into the limited space surrounding the machine. A set of co-axial electromagnet coils are at either end of the Pyrex section and provide a steady mirror field with mid-plane strength to $400 \mathrm{G}$ and mirror ratio to 60 .

The current-drive mechanism in this unique device is not definitively known. It may be due to $J \times B$ forces acting on electrons, in a manner similar to an induction motor, or to the azimuthal electric field created by the $\mathrm{RMF}_{\mathrm{o}}$ near the FRC's O-point null or to the diamagnetic effect or to any of several other mechanisms. Efficacy of these mechanisms depends on the penetration of the RMF into the $4-\mathrm{cm}$ radius plasma column. The collisional skin depth of PFRC plasmas is about $0.1 \mathrm{~mm}$ at $14 \mathrm{MHz}$.

\section{RF SYSTEM DESCRIPTION}

The odd-parity RMF is created by two sets of field coils with axes oriented at 90 degrees. These two sets are designated as North/South (N/S), and Top/Bottom (T/B). Each set consists of two coils, with the vacuum vessel in between. Each coil of a set is connected by an equal length of coaxial cable to a common (capacitive seriesparallel) matching network. A 90-degree phase difference is maintained between the N/S and T/B RF currents.

The RF drive source is a Stanford Research Systems DS345 function generator, which is amplitude modulated by a second SRS DS345 to produce pulses of several millisecond duration. In order to prevent thermal damage to components that must conduct high circulating currents, the duty cycle is generally fixed at $0.5 \%$. (Short term operation has been performed at $5 \%$ duty factor with pulses as long as $100 \mathrm{~ms}$.) A low level of RF power, ca., $100 \mathrm{~W}$, is provided between high power pulses, to sustain a weak plasma and hence to effect highly reproducible plasma density rise.

The SRS DS345 output feeds a high-gain solid-state amplifier, with maximum output of 40 Watts. This is fed to a Henry Radio $2 \mathrm{KD}$ amplifier, which boosts the power to up to 1200 Watts. The output of the $2 \mathrm{KD}$ is then fed to a hybrid splitter, the output of which is two RF signals of equal power, differing in phase by 90 degrees. A phase shifter is used on one output to compensate any phase offset introduced by the following amplifier stage. Each signal then drives a Henry Radio $8 \mathrm{~K}$ amplifier, each with an output up to 6000 Watts. Directional couplers are used to monitor each $8 \mathrm{~K}$ 's output, each of which is fed into a separate matching network. The RMF coils of each set are connected by equal lengths of coaxial cable to the matching network, where they are connected in parallel, and matched to the $50 \mathrm{Ohm}$ output of the Henry Radio 8K amplifier.

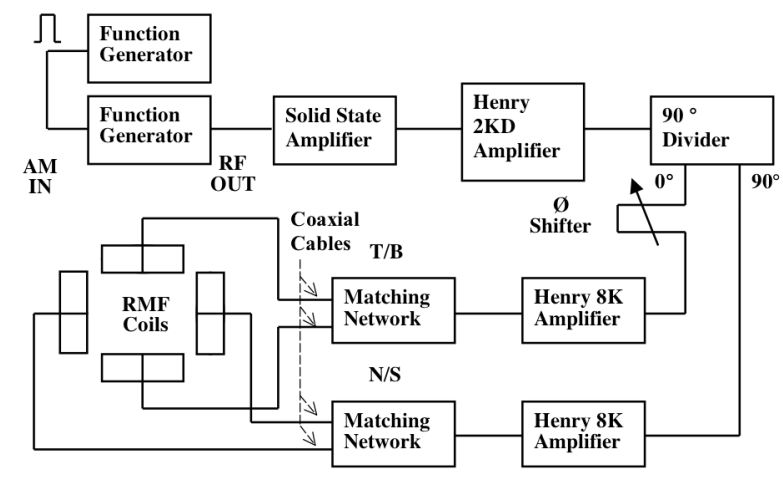

Figure 1. RF System Block Diagram

\footnotetext{
* Work supported by DOE Contract DE-AC02-76-CH03073
} 
The choice of RF amplifiers was limited by budget, reliability, and safety concerns. The Henry Radio amplifiers are tuned output, with bandwidths of several hundred KHz. Some experiments call for varying the frequency, so a tuned amplifier is not the ideal choice, however broadband amplifiers are considerably more expensive. The Henry Radio amplifiers are a reasonable compromise of price $v s$. power output and bandwith.

The configuration of a single RMF coil is shown in Fig. 2. The salient feature is the center RF feed which is necessary to create an odd-parity field. The design of the RMF coils and the matching network is critical to the efficient use of the available RF power. The goal is to obtain the reqired currents in the RMF coils with the available power, and to deposit power in the plasma, for driving current and heating. Plasma loading is only several tenths of an ohm in series with each RMF coil, so losses in the high current paths must be minimized. Each coil consists of two parallel branches with a common feed. Rogowski coils are used to monitor the currents in each branch of the coil. A 1-cm wide ground plane lies between the RMF coil and the vacuum vessel, and is connected to the electrostatic shields of the Rogowski coils. The purpose of the ground plane is to reduce electrostatic coupling to the plasma. The ground plane is broken so as not to form a continuous loop within the RMF.

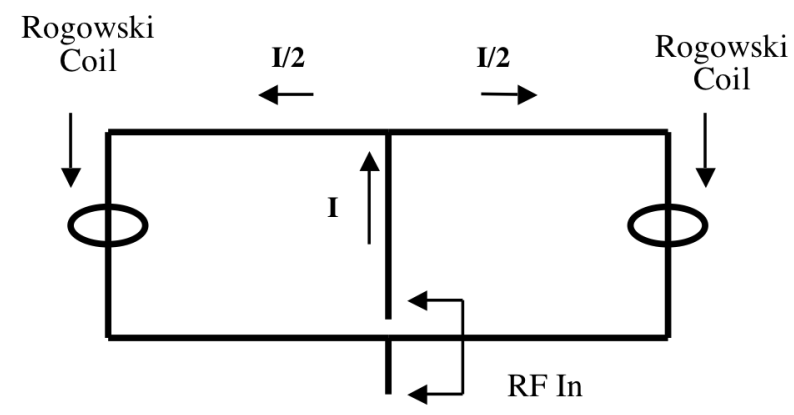

Figure 2. Single-turn RMF Coil schematic. The central feed of the coil, resulting in oppositely directed radial magnetic fields to the left and right, is the defining feature of odd parity.

The RF current from the matching network is equally divided between the two parallel branches of each coil, producing radial magnetic fields of equal magnitude, but opposite direction. The arrangement is such that there should be no mutual coupling between the N/S and T/B coil sets. Proper functioning of the FRC experiment is predicted to depend on the currents being closely balanced - to $\pm 1 \%$ - in all RMF coils. Figure 3 shows radial and axial $\mathrm{RMF}_{\mathrm{o}}$ amplitudes as a function of axial position, measure by an axially scanning two-axis $(\mathrm{r}, \mathrm{z})$ magnetic pick-up sensor. For this measurement, only the
T/B coils were powered and the scanning probe was located $2 \mathrm{~cm}$ off the major axis. (The short wavelength oscillations in the $B_{r}$ signal are due to aliasing in the digital data storage system.) The direction of $B_{r}$ reverses at $\mathrm{z}=0$; the direction of $\mathrm{B}_{\mathrm{z}}$ reverses at $-7 \mathrm{~cm}=\mathrm{z}=7 \mathrm{~cm}$. Both the measured fields and Rogowski-measured antenna currents showed a balance near $\pm 1 \%$. The phase of the N/S to T/B antennas can be controlled to better than $\pm 0.5^{\circ}$.

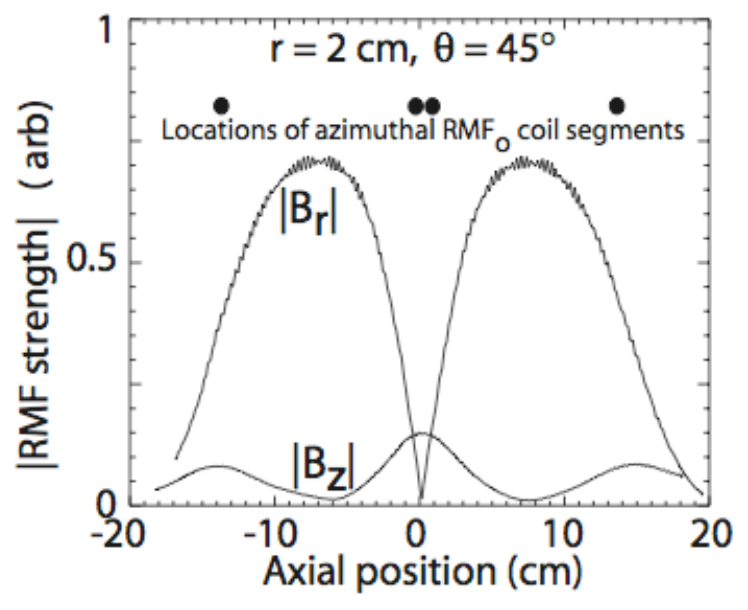

Figure 3. Measured RMF amplitudes.

\section{SYSTEM EVOLUTION}

\section{A. Long-transmission-line configuration}

In the first series of experiments, single-turn solid conductor RMF coils with an inductance of $150 \mathrm{nH}$ were used. These were fed by 230-cm-long RG 214 cables whose length was the dominant factor in obtaining a low antenna resonant frequency, near $7 \mathrm{MHz}$. It was found that too high a duty cycle would heat the center conductor of the RG 214, partially melting the polyethylene dielectric. To avoid this, it was decided to fix the duty cycle at $0.5 \%$. In addition, a simple circuit was built that will shut off the RF drive in the event that the average power exceeds a user-set threshold. The circuit Q without plasma is an indicator of system losses, and was found to be approximately 45 , less than anticipated and needed. In addition, there was a high level of mutual coupling between the coil sets. The nature of this coupling was to add to the current in one RMF coil set, and subtract in the other, resulting in significant imbalances.

\section{B. Short transmission line configuration}

In the next configuration, a shorter, lower loss transmission line and two-turn RMF coil of silver-plated 6-mm-OD copper tubing were used. The additional turn was added to increase the strength of the RMF. The inductance was $500 \mathrm{nH}$. The RFCs were constructed with a one-diameter space between the turns, chosen to 
increase turn-to-turn voltage standoff, and to minimize losses due to the "proximity effect"[4]. Proximity effect is the rise in RF resistance due to non-uniform current distribution in closely spaced conductors. Silver plating reduces losses at contacting surfaces. RG 214 proved to be a poor choice. It is a relatively small $(2.3 \mathrm{~mm}$ diameter inner conductor) cable, with a stranded inner conductor. Stranded conductors have a higher RF resistance than solid conductors of equal diameter. A shorter length (36 $\mathrm{cm})$ of RG 217 cable connects the RFC to the matching network. The shorter length results in a lower value of transformed inductance at the matching network. This allowed operation at higher RMF frequency, hence access to lower density, higher temperature plasma regimes. (Using the matching network, the operating frequency in this configuration could be tuned between 10 and 20 MHz.) RG 217 has a solid inner conductor $30 \%$ larger in diameter than RG 214. Losses are lower as a result, but the cable is less flexible. In addition, some stranded conductors in the matching network were replaced with solid copper. The Q now had a value of 135. Several factors contributed to this improved circuit $\mathrm{Q}$ :

- The reduced losses in the transmission lines.

- The two-turn RMF coil has twice the loss, but greater than three times the inductance of a single-turn coil.

- The ratio of RMF coil reactance to RF resistance is proportional to the square root of the frequency.

One consequence of higher $\mathrm{Q}$ is higher voltage for the same power. Adequate clearances at the cable ends are required to assure adequate voltage standoff. The mutual coupling previously encountered was found to be due to $\mathrm{RF}$ currents running on the outside of the cables connecting the matching network to the $8 \mathrm{~K}$ Henry amplifier. These currents were suppressed by the addition of ferrite toroids around the cables where they exit the matching network enclosure.

\section{RF AS A PLASMA DIAGNOSTIC}

Though the primary functions of the RF rotating magnetic field are current drive and heating, it can also supply information concerning plasma behavior.

\section{A. RMF Penetration into the plasma}

Deep penetration of the RMF into the plasma is essential to obtain the best heating and current drive. Theory for even-parity RMF[5] states that penetration is a function of the relative rotational frequency of the magnetic field and the electron velocity associated with the azimuthal current. When electrons travel synchronously with the RMF, the relative frequency is zero, and penetration is complete. Collisions are one phenomenon that decrease the azimuthal electron flow speed.

If the RMF does not penetrate into the plasma, the inductance of the RMF coils is reduced and the resonant frequency, $\mathrm{f}_{\mathrm{r}}$, of the RF network rises. This will be indicated by an increase in the reflection coefficient at the input to the matching network. The frequency of the RF signal generator may be shifted upwards until the reflection coefficient has reached a minimum, and resonance has been re-established. The amount of frequency shift may be used to calculate the change of inductance and is thus a quantitative measure of the RMF penetration distance. When the resonant frequency under plasma conditions is the same as under vacuum, complete field penetration is indicated. Figure 4 shows the measured shift in $f_{r}$ caused by the insertion of copper pipes along the PFRC axis. This graph serves as the calibration connecting frequency shift to RMF penetration. The measured shifts are close to the expected values, proportional to the pipe radius squared.

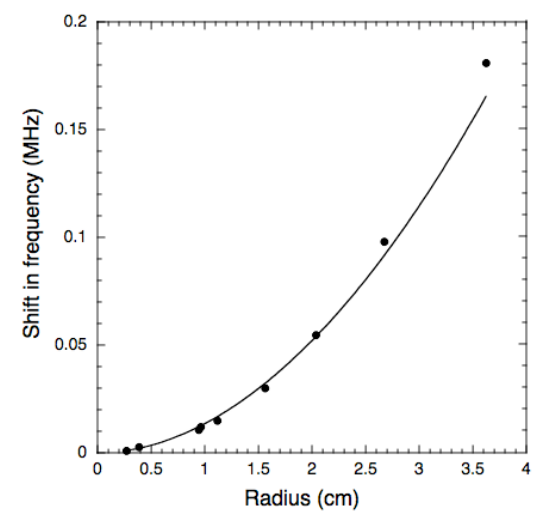

Figure 4. Shift in resonant frequency, $f_{r}$, caused by the insertion of copper pipes on the axis of the PFRC. With no pipes inserted, $\mathrm{f}_{\mathrm{r}}=14.0044 \mathrm{MHz}$. The curve shows the best fit to $r^{2}$.

\section{B. Plasma Loading}

It is desirable to know what the plasma loading is, and how much power is deposited in the plasma. The power delivered to the plasma is estimated by accounting for all power input to the matching network. This power is distributed as losses among circuit elements and transmission lines, with the remainder going to the plasma.

The first step is to create a circuit model of the matching network and rotating field coils. Values of $\mathrm{C}_{\mathrm{S}}$ and $\mathrm{C}_{\mathrm{P}}$ are chosen to provide an impedance of $50+\mathrm{j} 0$ Ohms at RF IN, see figure 5, under vacuum loading. The bandwidth, which is determined by the total losses in the system, is measured under vacuum. The circuit model is constructed to match this measured bandwidth. Some components, such as transmission lines, have circuit parameters that are available from the manufacturers. 
Losses in the RFCs are determined by such factors as skin depth and proximity effects. In addition, there will be stray losses distributed among the various connections. Stray losses account for approximately $20 \%$ of the net power. The matching network must handle the combined currents of both RMF coils in a set, so the highest losses are found here.

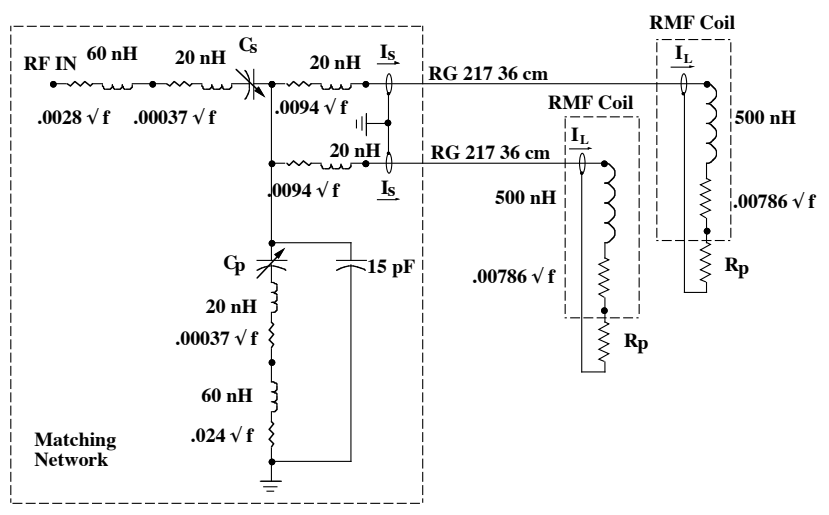

Figure 5. RF System Circuit Model

Consistent with the Rogowski coil measurements, it is assumed that the power is split equally between the two RG217 cables, and the currents in the RFCs are equal. Each RFC consists of two mutually coupled parallel inductances with a common feed point. Rogowski coils are located on each of the parallel inductances. The load current, $I_{L}$, is the sum of the currents in the parallel inductances. The average value of the four Rogowski signals is used to determine $\mathrm{I}_{\mathrm{L}}$. The load impedance $\mathrm{Zl}$ is the series combination of RMF coil impedance, and $R_{P}$, plasma loading. The impedance transformation by the transmission line is calculated, as well as the voltage and current $\left(I_{S}\right)$ at the source end. $I_{S}$ is used to calculate voltages, currents and losses in the rest of the matching network.

The Rogowski coils are used to compare the relative loading change from vacuum (no plasma) to plasma. Power is applied to the system under vacuum, net power and the currents in the rotating field coils are measured. A calibration factor is applied to the Rogowski signals to obtain the currents that result in the same total power in the model.

Plasma loading is determined by measuring the net power into the matching network and RMF coil currents when plasma is present. The calibration factor determined above is applied to the Rogowski signals, and average RMF coil currents are obtained. An arbitrary value of plasma resistance $\mathrm{Rp}$ is assigned, and the $\mathrm{RF}$ power delivered to the plasma is $\mathrm{I}_{\mathrm{L}}{ }^{2} \mathrm{Rp}$. The currents and dissipated powers are then calculated for the rest of the circuit. The value of plasma resistance is varied until the sum of all powers calculated is equal to the net input power measured. Table 1 shows typical values of loading and circuit losses.

Table 1. Typical Operating Parameters

\begin{tabular}{|l|l|l|}
\hline & $\mathrm{N} / \mathrm{S}$ & $\mathrm{T} / \mathrm{B}$ \\
\hline Net Input Power & $5496 \mathrm{~W}$ & $5463 \mathrm{~W}$ \\
\hline Plasma & $3094 \mathrm{~W}$ & $3154 \mathrm{~W}$ \\
\hline Matching Net. & $1220 \mathrm{~W}$ & $1168 \mathrm{~W}$ \\
\hline Cables & $872 \mathrm{~W}$ & $839 \mathrm{~W}$ \\
\hline RMF Coils & $310 \mathrm{~W}$ & $298 \mathrm{~W}$ \\
\hline Rp & $.295 \Omega$ & $.312 \Omega$ \\
\hline Il & 72 Arms & 71 Arms \\
\hline
\end{tabular}

\section{CONCLUSION AND PLANS}

The RF system has operated at combined powers of 11 $\mathrm{KW}$ at $14 \mathrm{MHz}$, with up to $7 \mathrm{~kW}$ delivered to the plasma. Full penetration of the RMF has been observed as well as electron temperatures of $220 \mathrm{ev[3].}$

Future plans call for the use of four 8k Henry amplifiers operated in pairs. The outputs of each pair will be combined to drive an RMF coil set. Another possibility is to combine all four $8 \mathrm{~K}$ amplifier outputs, and to split the power with a ninety-degree hybrid coupler. In this configuration the power balance is no longer dependant on the amplifiers having equal gain, and the hybrid will isolate the amplifiers from variations in the load.

\section{REFERENCES}

[1] S.A. Cohen and R.M. Milroy, "Maintaining the closed magnetic-field-line topology of a field-reversed configuration with the addition of static transverse magnetic fields," Phys. Plasmas 7, 2539 (2000).

[2] A.H. Glasser and S.A. Cohen, "Ion and electron acceleration in the field-reversed configuration by oddparity rotating magnetic fields," Phys. Plasmas 9, 2093 (2002).

[3] S.A Cohen, B. Berlinger, C. Brunkhorst, A. Brooks, N. Ferraro, D.P. Lundberg, A. Roach, and A.H. Glasser, "Formation of Collisionles High- $\beta$ Plasma by Odd-Parity Rotating Magnetic Fields", Phys. Rev. Lett. 98, 145002 (2007).

[4] F. E. Terman, "Radio Engineers Handbook", New York: McGraw Hill, 1943, pp 36.

[5] W.N. Hugrass and R.C. Grimm, "A numerical study of the generation of an azimuthal current in a plasma cylinder using a transverse rotating magnetic field," J. Plasma Physics 26, 445 (1981). 